\title{
Comparative study of two 3D reconstruction methods for underwater archaeology
}

\author{
Arnaud Meline, Jean Triboulet and Bruno Jouvencel
}

\begin{abstract}
The underwater 3D reconstruction cartography has made great progress in the last decade. The work presented in this paper is about the analysis and $3 \mathrm{D}$ reconstruction of archeological objects. Using a calibrated single camera and an uncalibrated system, we propose to describe a method to perform the Euclidian 3D reconstruction of unknown objects. A comparison of two methods is presented and tested on synthetic and real underwater pictures. Filters are proposed to simulate underwater environment and inherent problems. Finally, robust and stable features have been extracted from underwater pictures and used to perform the 3D model.
\end{abstract}

\section{INTRODUCTION}

In this paper, our aim is to analyze natural underwater scenes and especially the 3D cartography of submarine environments. To handle this problem, several techniques are widely used today. The originality of our project involves the fusion of two kinds of maps obtained with sensors of different resolutions. An Autonomous Underwater Vehicle analyzes the seabed with a lateral sonar to construct a first global map of the zone (fluvial archaeology or coastal oceanographic applications). This map is then decomposed into smaller cells representing a mosaic of the seabed. A second scanning is performed on particular cells using a second sensor with a higher resolution leading to a detailed 3D partial map. In our case, we aim at applying this method to submerged archaeological sites, where objects of interest (statues, plates, amphora) are detected in the global map, while 3D precise reconstruction would be necessary. This project includes two parts: the first part correspond to the 3D reconstruction of constrained submarine environment with a video camera. The Second part is the multimodal aspect of the problem. The work presented in this paper focuses on the first part of the project and deals with the 3D reconstruction of the submarine environment with a video camera.

Nowadays, even if vision can solve many problems, images analysis in case of submarine environments is quite complicated. The different methods used on the surface are not robust enough to changes produced by the underwater medium. So, the robustness of several methods decreases and their results become unstable.

When a scene or an object must be reconstructed in $3 \mathrm{D}$, detection and matching points in the image are crucial parts for the accuracy of the model. If these steps contain errors, the 3D model will be of insufficient quality or completely wrong. Thus, we used a well known methodology

A. Meline, J.Triboulet and B. Jouvencel are with LIRMM, Univ. Montpellier 2 - CNRS, 161 rue Ada, 34392 Montpellier, France \{meline, triboulet, jouvencel\}@lirmm.fr

This work is supported thanks to ANR project CFLAM and UNIMES. for $3 \mathrm{D}$ reconstruction and we have tested and compared the robustness of the algorithms which are sensitive to underwater disturbances. We propose to use surface images on which are applied filters simulating underwater characteristics. Therefore, we can recreate the underwater conditions and determine which setting defeats the reference methods used on the surface. From these results, we selected the best methods to achieve the 3D model of a submerged object.

This paper is organized as follows. Section II presents a survey on 3D reconstruction and problems caused by the underwater environment. In section III, a method to reconstruct underwater 3D scene is presented. Section IV describes the detector and the matching methods. In section V, the filters employed to simulate underwater characteristics are detailed. Section VI presents experimentations results: the comparison of detector methods used and the 3D reconstruction. Finally, conclusion and future work are presented in section VII.

\section{RELATED WORKS}

In recent years, the community of computer vision widely studied the topic of 3D reconstruction from image sequences. Numerous methods exist, but they cannot be applied to all underwater images. However, they depend on the knowledge of the environment and the used system. For example, the work of Snavely [1], Bartoli [2] and Barazzetti [3] using unknown images to create 3D urban models. They have no information about the devices that took the pictures but their methods utilize a priori knowledge of the scene. By focusing on underwater reconstructions, we find the work of Brandou [4] who proposes to calibrate the camera in situ and to use a robot arm to move it. With this system, he knows the exact camera movement and simplifies the reconstruction. Espiau [5] works on the same images but proposes a method to reconstruct textured scenes from an uncalibrated camera. To achieve this problem, he proposes to use the scene rigidity to estimate the camera parameters. In a different way, Kim [6] proposes a SLAM approach to inspect the ship hull status. He determines the camera position in 3D using a calibrated monocular camera and a geometric model selection. Unlike Beall [7] or Negahdaripour [8] who uses a full calibrated stereo rig to pre-built the 3D trajectory or to inspect ship hull. Then, he simultaneously optimizes the camera trajectory and estimates a 3D dense reconstruction. In a same way, Hogue [9] combines a 3DOF inertial sensor and a calibrated stereo ring to estimate the trajectory and creates 3D dense map.

All these applications impose constraints (type of scene, knowledge of the camera movement, calibration parameters, shape of the object) which change the approach. Some 
authors, like Hartley [10], Pratt [11], Szeliski [12] and Nicosevici [13] propose general methods to reconstruct a 3D scene. To obtain Euclidian structure of scenes, it is fundamental to know the intrinsic camera calibration parameters. Many methods exist to estimate these parameters on the surface but when the camera is in a submarine environment, it introduces optical problems. To avoid these problems, Pessel [14] proposed a self-calibration method. Other authors like Lavest and Rives [15] proposed to adjust the surface parameters to the underwater environment. We [16] proposed another approach that includes the environment change in the radial distortion model.

All the reconstruction methods need robust feature points to obtain the best 3D model. So the feature points detection and matching is a crucial step for $3 \mathrm{D}$ reconstruction. There are many detection points methods [12] such as Moravec corner detector, Kitchen and Rosenfeld detector, Forstner operator, Hessian detector, SURF, Ferns, Harris and Stephens detector, SIFT... All of these feature detectors have invariant characteristics in a spatial domain. But they have lacking quality results when images undergo important modifications like underwater images. Indeed, the presence of suspended particles, the energy absorbing of light ray and the variation of water refractive index make blur and add noise on images. All methods are not robust enough for such modifications. In next parts, we will compare a robust method (SIFT) with respect to a classical one (Harris and correlation).

\section{3D RECONSTRUCTION METHOD}

As the geometry of the scene and the camera motion are unknown, we used the method proposed by Hartley and Zisserman [10] to achieve 3D reconstruction. Fig. 1 resumes the different steps to obtain the 3D model. Each block will be detailed.

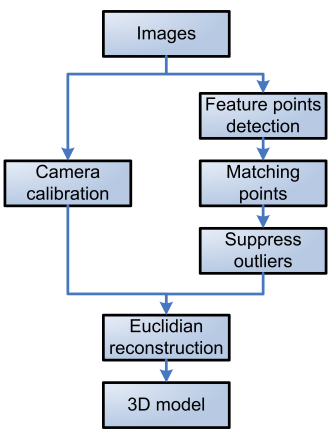

Fig. 1: 3D reconstruction diagram

\section{A. Camera calibration}

The goal of our problem is to obtain an Euclidian reconstruction, so we have to calibrate our camera. To take into account the underwater parameters which affect the precision, we used the method presented in [16]. We proposed to include the underwater constraints in the camera distortion model. Thus, we used a pinhole model taking into account radial distortion terms. Then calibration is done using the Zhang's method [17]. The major advantage of this method is the utilization of a simple planar calibration target and the integration of underwater optical constraints.

\section{B. Feature points detection and matching}

As we saw in section II, the detection and matching of the feature points is crucial to obtain a representative 3D model of the observed scene. In this paper, Harris and SIFT method are used to obtain matching points and fundamental matrix. The motivation of those choices and detailed methods will be presented in section IV.

\section{Euclidian Reconstruction}

To perform the reconstruction, we use the essential matrix method proposed by [10]. The purpose of this method is to obtain the pose of the second camera with respect to the first one. We define the following projection matrix $P 1=K[I \mid 0]$ for the first camera and $P 2=K[R \mid t]$ for the second. Then, we estimate the rotation matrix $(R)$ and the translation vector $(t)$. The essential matrix $E=K^{t} F K$ is calculated from fundamental $(F)$ and calibration $(K)$ matrices obtained previously. Then, calculating the Single Value Decomposition (SVD) of the essential matrix gives us the camera pose $(R$ and $t)$. Finally, the $3 \mathrm{D}$ reconstruction is done by a linear triangulation from the inliers found earlier and the projection matrices.

The 3D model is then treated to remove some aberrant points that are locally isolated. The mesh structure is achieved through a 3D Delaunay triangulation.

\section{Detection And MATChing}

This phase is divided in two parts. The first part shows how to extract feature points on images and the second part present how to realize the matching between all detected points and check if all matching points are correct using the epipolar geometry constraints. In this section, we present the two methods used to detect and match points, then we detail the verification and the removal of false matching (outliers).

\section{A. Features points detection and matching}

The feature points detection and matching is an essential step for $3 \mathrm{D}$ reconstruction. The quality of the $3 \mathrm{D}$ model is related to the precision of these elements.

There are many detection point methods but they do not have the same performances. Indeed, Harris can find points on objects, more specifically near the corner. This enables us to obtain the features points of the object's geometry.

SIFT is considered as one of the best performing detectors in many applications because of its robustness to scaling, rotations, translations and illumination changes. We decided to compare these two representative methods in our work.

1) Method 1: Harris detector and correlation matching: Harris and Stephens [18] proposed an operator to detect corners in image. They used a criterion based on corners and edges informations by calculating the image derivative along $\mathrm{x}$ and $\mathrm{y}$. The values of this criterion is positive near a corner, negative near an edge and low in a region of constant intensity. The detected points are selected by choosing the highest values.

To match these points, we used a Normalized Cross Correlation (NCC) method. It consists in searching the corresponding points in the other image which have a maximum 
of correlation value. To improve performance and reduce the computational time, we used a guiding approach for this matching. A method detecting the global motion between the two images is used to reduce the number of candidates to selected points. Then, only pairs of corresponding points in both directions are matched and one point can only have a single matched point in the other image. Thus we limit the selection of false matches.

2) Method 2: Scale Invariant Feature Transform (SIFT): The SIFT method [19] extracts interest points after series of treatments (pyramid of images, Differences of Gaussian, finding extrema). Then each point becomes a Keypoint by assigning a descriptor vector with 128 dimensions. This invariant vector represents the gradient norm for eight different orientations in each zone of a $4 \times 4$ window around the point. This vector is then used to associate a point in the first image with one of the points of the second image. The matching is done with an Euclidian distance calculus.

\section{B. Suppress false matching with the epipolar geometry}

Previous methods present some limits and can therefore provide false matches ("outliers"). To reinforce this step, we used the epipolar constraint to verify all the matching points and calculate the fundamental matrix.

The RANSAC algorithm [20] can classify the matched data into good and false matches (inliers and outliers) using the calculation of the fundamental matrix. The algorithm select randomly eight points and estimate the fundamental matrix from the "eight points method" presented by Hartley in [21]. Then, the distance between the projected point in the second image and the epipolar line is calculated for each point. If this distance exceeds a threshold, the point is rejected and the remaining items are grouped in a set. The process is repeated and the set with the maximum of elements is selected. Finally, the fundamental matrix is estimated with these points.

Once the fundamental matrix estimated and the inliers obtained, the movement between two successive images can be deduced (e.g. section III-C).

\section{UNDERWATER ENVIRONMENT PROBLEMS AND CORRESPONDING FILTERING}

The work presented in [16] demonstrates that under water, Harris detector with correlation gives better results than SIFT. However, for the vision community, SIFT is one of the most efficient detector in many applications. Comparisons have been performed on surface images but few for underwater pictures [22], [23].

In order to find which underwater parameters have failed SIFT algorithm, we propose to take surface picture and add noise filters that represent each of these disturbances.

Water introduced variables effects on the signal propagation decreasing the images quality. The main causes of this degradation is due to the refractive index, the presence of particles and the energy absorption of light ray according to their wavelength (color loss).
Some authors like [24], [25] have created global model more or less detailed of these disturbances, but none has decomposed every phenomena. The use of these filters do not permit us to determine what decreases SIFT performances.

Presence of suspended particles (turbidity) causes refraction and reflection phenomena on light rays. To model this phenomenon, we proposed to use a speckle noise. Indeed, this effect is often studied by physicist who characterize the suspended particles from the speckle noise. They used an image of the speckle noise generated by the underwater environment to determine some particles characteristics likes velocity, density, etc... [26], [27]. Therefore, turbidity can be represented by a speckle noise.

In an other hand, the changing of the refractive index introduces a blur effect and decreases the dynamic components on the images. To recreate this effect many filters can fit, however we chose to use a Gaussian low pass filter [28].

Regarding the energy absorption of light ray, as depth increases, colors drop off one by one depending on their wavelength. Low frequency color like red disappears at first $\sim 3 \mathrm{~m}$. Then orange color is lost $\sim 5 \mathrm{~m}$. Blue color travels the longest in water due to its higher frequency. To model this phenomenon, an attenuation on the amplitude of colors channels is used.

\section{EXPERIMENTATIONS AND RESULTS}

\section{A. Underwater experimentations}

A camera/camcorder was used for these experiments. The resolution used for pictures extracted from the video is $1280 \times 720$ pixels. A waterproof case fitted the device to take underwater pictures obtained at sea in shallow water $(15 \mathrm{~m})$. We immersed various objects (a statue of a female bust, a statue of a fish, bowls, plates and a calibration grid) which we are able to compare with 3D scanned models. We also took pictures of the same objects in a pool to limit some noises and in surface to apply filters and simulate underwater pictures. The results presented in this document are principally made with images of the female bust. In this experiment, different pictures were taken in order to cover all the object.

\section{B. Detection and matching results}

As we have seen previously, the detection and matching step is an essential point of the $3 \mathrm{D}$ reconstruction. Thus, the two methods presented in Section IV have been examined and compared in different situations.

\section{1) Sea environment:}

Initially, we tested these methods on sea images in order to determine the best method for this kind of pictures. Table I summarizes the results obtained from four pairs of images. The first three pairs of images are selected to have a small displacement. The last image pair shows a greater movement. The detections thresholds are adjusted to get roughly the same number of feature points. For each pair, we compared: the number of feature points detected in both images, the number of inliers and the inliers percentage.

These experiments show that Harris method gives better results and more points (17\% more) than the SIFT algorithm, 
TABLE I: Comparison of inliers number obtained with the two methods in sea environment

\begin{tabular}{|c|c|c|c|c|}
\hline & \multicolumn{2}{|c|}{ Harris } & \multicolumn{2}{c|}{ SIFT } \\
\cline { 2 - 5 } & $\begin{array}{c}\text { Detected } \\
\text { points }\end{array}$ & Inliers & $\begin{array}{c}\text { Detected } \\
\text { points }\end{array}$ & Inliers \\
\hline \multirow{2}{*}{ Pair 1 } & $902 \mathrm{I} 1$ & 305 & $902 \mathrm{I} 1$ & 162 \\
& $902 \mathrm{I} 2$ & $34 \%$ & $1122 \mathrm{I} 2$ & $18 \%$ \\
\hline \multirow{2}{*}{ Pair 2 } & $797 \mathrm{I} 1$ & 244 & $797 \mathrm{I} 1$ & 146 \\
& $797 \mathrm{I} 2$ & $30 \%$ & $894 \mathrm{I} 2$ & $18 \%$ \\
\hline \multirow{2}{*}{ Pair 3 } & $894 \mathrm{I} 1$ & 310 & $894 \mathrm{I} 1$ & 148 \\
& $894 \mathrm{I} 2$ & $35 \%$ & $999 \mathrm{I} 2$ & $17 \%$ \\
\hline \multirow{2}{*}{ Pair 4 } & $1148 \mathrm{I} 1$ & 291 & $1505 \mathrm{I} 1$ & 186 \\
& $1148 \mathrm{I} 2$ & $26 \%$ & $1148 \mathrm{I} 2$ & $16 \%$ \\
\hline
\end{tabular}

contrary to what is usually found for surface images. In case of larger movements, the results are decreasing because we reach the limits of the correlation method which is valid only for small displacement.

\section{2) Surface images and filtering:}

The SIFT method does not work well under water, so we proposed to find which characteristic of the underwater environment is problematic. We used surface images on which proposed filters (Section V) are added to simulate underwater conditions. Both detection methods are tested with only one filter in a first time, then all filters are added to create an synthetic underwater image.

Fig. 2 represents the colors histogram of a sea, a surface and a filtered image. The first filter used to test these methods simulates the water color absorption phenomenon. To determine the proportion of color attenuation, a colors histogram of sea and surface images was studied. The red plane has completely disappeared in the sea picture. However, green and blue planes have suffered a decrease of $15 \%$ and $13 \%$ with respect to the surface image. Others pictures were compared and the same results were obtained. These results are consistent with theoretical attenuations at this depth $(\sim 15 m)$. Indeed, the red color disappears completely around $3 \mathrm{~m}$ and $5 \mathrm{~m}$ and the green and blue colors are less reduced. For this experimentation, images were taken in the same orientation and resolution.

The parameters are used to adjust the color attenuation filter on all pictures. Fig. 3 represents the sea image (a) and the obtained image (b) with the color filter (the color is roughly the same between these two pictures).

Afterwards, we identified the variance of the blur and the speckle in the sea images. These two noises are estimated together for two reasons. First, these noises are physically linked. Second, measurement and exact identification of the core noise in the images are not achievable accurately. Therefore, we proposed to calculate the local variance of images and determine simultaneously the parameters of the Gaussian and speckle filter. We analyzed the histogram and calculated the average of the local variance of the sea image. Then, we adjusted the filter parameters to obtain the same value of variance between the sea and the filtered images. We have found a variance of 0.02 for the speckle noise and a variance of 8 for a windows size of $[9 \times 9]$ for the Gaussian filter. Fig. 4 shows the results achieved for sea, surface and filtered image with the parameters determined above from

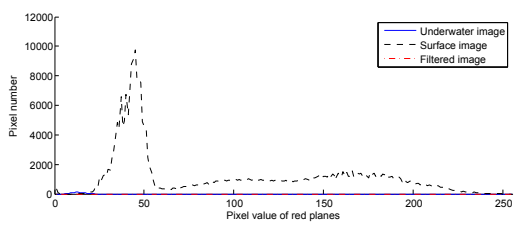

(a) Red histograms

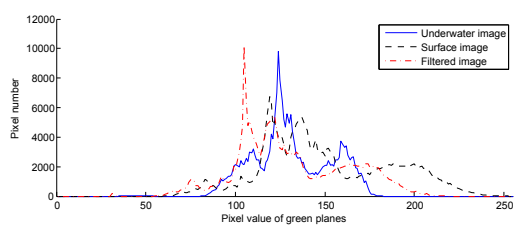

(b) Green histograms

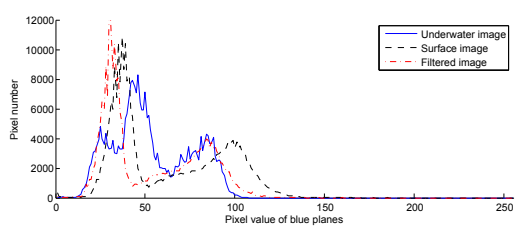

(c) Blue histograms

Fig. 2: Histogram of red (a), green (b) and blue (c) planes of sea, surface and filtered picture

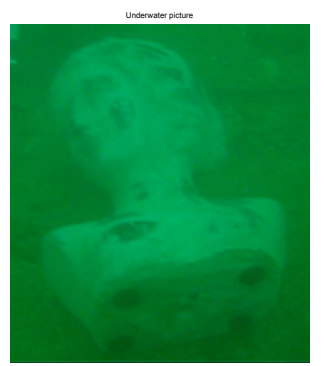

(a) Sea picture

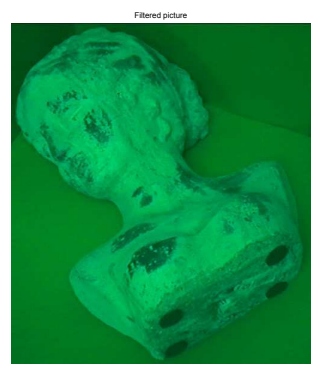

(b) Filtered picture
Fig. 3: Sea picture (a) and surface picture filtered with color filter (b)

the local variance histogram.

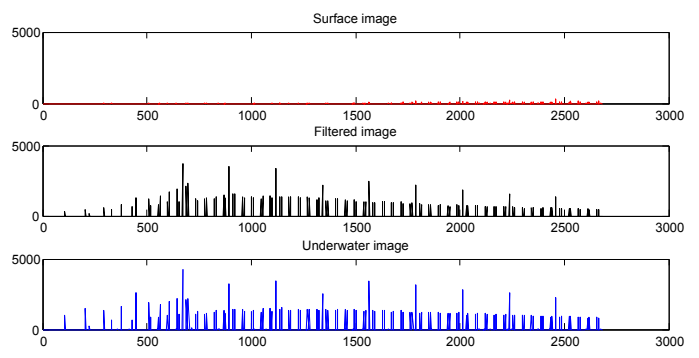

Fig. 4: Histogram of a surface, a filtered and a sea image.

The parameters of both filters defined, we have applied them to the surface image and obtained an image almost identical to the sea image (Fig. 5).

After the filter estimation, their effects were tested on the two methods of detection/matching points. At first, we used two images whose displacement corresponds to the fourth pair of images used in table I. Table II summarizes the results achieved for the different methods according to the applied filters.

As these experiments show, SIFT gives better results than Harris in similar situation to the surface such as blur or color 


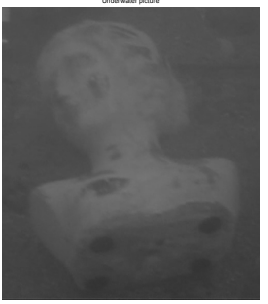

(a) Sea picture

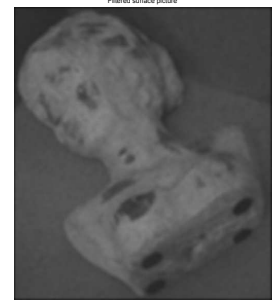

(b) Filtered picture
Fig. 5: Sea picture (a) and surface picture filtered with spekle and blur (b)

TABLE II: Comparison of the two methods with different filters applied to surface images

\begin{tabular}{|c|c|c|c|c|}
\hline & \multicolumn{2}{|c|}{ Harris } & \multicolumn{2}{|c|}{ SIFT } \\
\hline & $\begin{array}{c}\text { Detected } \\
\text { points }\end{array}$ & Inliers & $\begin{array}{l}\text { Detected } \\
\text { points }\end{array}$ & Inliers \\
\hline \multirow{2}{*}{ No one } & 617 I1 & 154 & $625 \mathrm{I1}$ & 212 \\
\hline & $617 \mathrm{I} 2$ & $25 \%$ & $617 \mathrm{I} 2$ & $35 \%$ \\
\hline \multirow{2}{*}{ Color } & 629 I1 & 143 & 692 I1 & 223 \\
\hline & $629 \mathrm{I} 2$ & $22 \%$ & $629 \mathrm{I} 2$ & $36 \%$ \\
\hline \multirow{2}{*}{ Blur } & $631 \mathrm{I1}$ & 140 & $631 \mathrm{I} 1$ & 227 \\
\hline & $631 \mathrm{I} 2$ & $22 \%$ & $666 \mathrm{I} 2$ & $36 \%$ \\
\hline \multirow{2}{*}{ Speckle } & 581 I1 & 139 & 581 I1 & 36 \\
\hline & $581 \mathrm{I} 2$ & $24 \%$ & $593 \mathrm{I} 2$ & $6 \%$ \\
\hline \multirow{2}{*}{$\begin{array}{c}\text { Speckle \& } \\
\text { Blur }\end{array}$} & 631 I1 & 152 & $631 \mathrm{I} 1$ & 91 \\
\hline & $631 \mathrm{I} 2$ & $24 \%$ & 641 I2 & $15 \%$ \\
\hline \multirow{2}{*}{ All } & 583 I1 & 153 & 608 I1 & 84 \\
\hline & $583 \mathrm{I} 2$ & $26 \%$ & $583 \mathrm{I} 2$ & $15 \%$ \\
\hline
\end{tabular}

change (due to a non natural lightning). But when speckle noise is added, the quality of results decreases dramatically with only $6 \%$ of inliers. This deterioration is due to the descriptor part of SIFT algorithm. The descriptor is based on oriented gradients. The speckle noise adds a "granularity" on images, which significantly disrupts the oriented gradients.

Subsequently, we tested different combinations of filters and we have seen that whenever the speckle noise is present in the image, SIFT results correspond to those that were expected from the results of sea pictures. We realized that Harris does not suffer practically any deterioration whatever the filters used. Inliers rate remains around $25 \%$ in all cases.

To check if this trend was general, we made the same experiments as before on a group of twenty images with different objects and backgrounds. The minimum, maximum and average of the two methods inliers percentages are computed in Table III.

TABLE III: Statistics of results of the two methods with different filters

\begin{tabular}{|c|c|c|c|c|c|c|}
\hline & \multicolumn{3}{|c|}{ Harris Inliers } & \multicolumn{3}{c|}{ SIFT Inliers } \\
\cline { 2 - 7 } & Min. & Max. & Mean & Min. & Max. & Mean \\
\hline No one & 22.3 & 27.6 & 24.8 & 34.4 & 37.3 & 35.5 \\
\hline Color & 22.7 & 26.6 & 24.1 & 33.6 & 38.2 & 35.7 \\
\hline Blur & 21.4 & 27 & 24.5 & 34.8 & 38.9 & 36.4 \\
\hline Speckle & 21.3 & 26.2 & 23.9 & 5.6 & 7 & 6.2 \\
\hline Speckle \& Blur & 23.1 & 26.7 & 24.6 & 13.8 & 15.3 & 14.5 \\
\hline All & 24.1 & 27 & 25.7 & 12.1 & 15 & 14.1 \\
\hline
\end{tabular}

We conclude that the descriptor of SIFT method is not robust to speckle noise and thus is not suitable for underwater pictures. Contrary to the Harris/correlation method which is robust to any noises present in the water.

\section{3) Pool environment:}

In section VI-B.2, we have highlighted the weaknesses of SIFT descriptors to the speckle noise. In this section, we propose to test the two algorithms on pool pictures. Indeed, pool images are subject to the same noise model as sea images but turbidity has almost zero in pool. This medium characteristic allow us to test the two methods in water with a low speckle noise.

We tested both algorithms on twenty pictures of each type and made statistics from results. Table IV regroups statistics of inliers percentage of pictures took in various environments. The air, filtered and sea statistics were obtained with twenty images.

TABLE IV: Statistics of results of the two methods in different conditions (surface, filtered, sea and pool)

\begin{tabular}{|c|c|c|c|c|c|c|}
\hline \multirow{2}{*}{} & \multicolumn{3}{|c|}{ Harris Inliers } & \multicolumn{3}{c|}{ SIFT Inliers } \\
\cline { 2 - 7 } & Min. & Max. & Mean & Min. & Max. & Mean \\
\hline Air & 22.3 & 27.6 & 24.8 & 34.4 & 37.3 & 35.5 \\
\hline Filtered & 24.1 & 27 & 25.7 & 12.1 & 15 & 14.1 \\
\hline Sea & 21.4 & 28.1 & 25.1 & 11.9 & 16.7 & 15.2 \\
\hline Pool & 23.8 & 28.5 & 26.1 & 26.2 & 30.7 & 28.4 \\
\hline
\end{tabular}

These results reinforce our idea that speckle noise disrupts the SIFT descriptor. Indeed, the SIFT inliers rate is higher in pool than in sea. This phenomenon is due to the low number of suspended particles in pool and therefore to a weak speckle noise. Results remain lower than surface because noise is still present in pool pictures.

\section{C. $3 D$ reconstruction results}

In this part, we applied the method described in Section III. The reconstruction is performed from two images selected from a video. The choice of the two consecutive images (Fig. 6) is made so that the displacement between images is neither too large nor too small to ensure sufficient movement and prevent loss of information on the object.

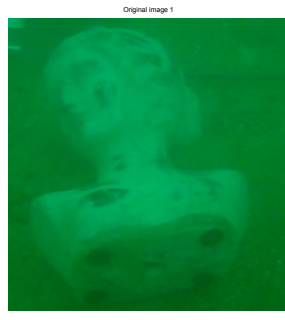

(a) Picture 1

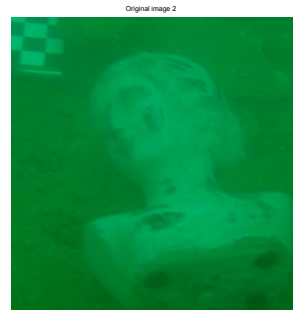

(b) Picture 2
Fig. 6: Underwater pictures used for 3D reconstruction.

As we demonstrated above, the method of Harris / correlation allows us to get more robust points in the image than SIFT. Therefore, the fundamental matrix estimation is realized from Harris points and the essential matrix is calculated. Finally, the transformation between two pictures is deduced. Fig. 7 represents the relative movement between two successive images deduced from matched points with Harris and SIFT methods.

However, SIFT points are not neglected. Even if the number of points is lower, those founded are mostly coherent and we can remove the last outliers through the epipolar constraint. These points are different from those found by 


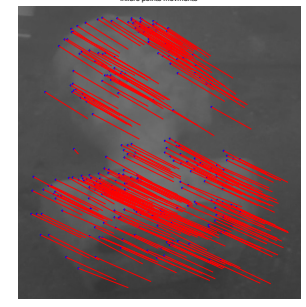

(a) Harris method

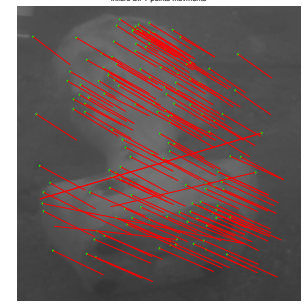

(b) SIFT method
Fig. 7: Feature points and estimate movement from Harris (a) and SIFT (b) method on the first image.

the Harris method, but they enable to increase the model resolution. On fig. 7, we can see a few numbers of outliers with the 2 methods. These points will create errors in the $3 \mathrm{D}$ models, but we can easily remove them because their representation in 3D is aberrant with respect to the structure. In general, they are isolated from the structure and thus easy to identify and remove.

To obtain the 3D model, a triangulation is performed using the inliers and the projection matrix found previously. Then, the mesh structure is achieved through a 3D Delaunay triangulation. Fig. 8 shows the 3D wireframe model.

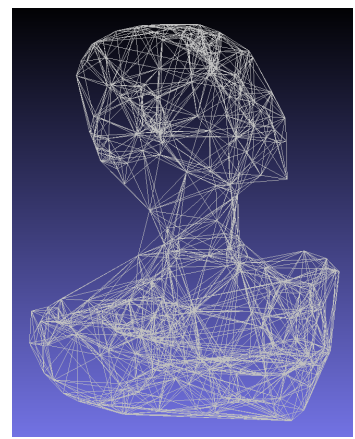

Fig. 8: 3D wireframe model.

\section{CONCLUSIONS AND FUTURE WORK}

The objective of this work is to obtain robust matching points to reconstruct $3 \mathrm{D}$ underwater archaeological objects with a single camcorder. To achieve this goal, we have to make sure that the feature points and matching are robust enough to underwater conditions. Three filters are determined and quantified to characterize the underwater environment (color, blur and speckle). We have drawn a parallel between two detecting/matching methods through various experiments which enable us to single out the most robust method. We concluded that the SIFT descriptor is not robust to speckle noise unlike Harris/correlation method. In the future, we plan to apply a despeckle filter on images and check if the performance of SIFT is increased.

Then we used an euclidian reconstruction method to create a 3D model from these robust points and camera parameters. The 3D model obtained is sparse because it is created from only two images, therefore we plan to enrich it with a $3 \mathrm{D}$ dense matching method. This will increase the accuracy of the $3 \mathrm{D}$ object model. Then, texture will be added on the model.

\section{REFERENCES}

[1] N. Snavely, S. M. Seitz, and R. Szeliski, "Photo tourism: exploring photo collections in 3d," ACM Trans. Graph., vol. 25, pp. 835-846, July 2006.

[2] A. Bartoli and P. Sturm, "Constrained structure and motion from multiple uncalibrated views of a piecewise planar scene," Int. J. Comput. Vision (IJCV), vol. 52, pp. 45-64, April 2003.

[3] L. Barazzetti, "A trip to rome: physical replicas of historical objects created in a fully automated way from photos," in the First int. conf. on Human-computer interaction, tourism and cultural heritage, HCITOCH'10, (Berlin, Heidelberg), pp. 63-72, 2011.

[4] V. Brandou, A. Allais, M. Perrier, E. Malis, P. Rives, J. Sarrazin, and P. Sarradin, "3d reconstruction of natural underwater scenes using the stereovision system iris," in OCEANS 2007 - Europe, pp. 1-6, 2007.

[5] P. Espiau, F.-X. Rives, "Extracting robust features and 3d reconstruction in underwater images," in OCEAN 2001, vol. 4, 2001.

[6] A. Kim and R. Eustice, "Pose-graph visual slam with geometric model selection for autonomous underwater ship hull inspection," in Proceedings of IROS'09, (Piscataway, USA), pp. 1559-1565, 2009.

[7] C. Beall, B. Lawrence, V. Ila, and F. Dellaert, "3d reconstruction of underwater structures," in Proceedings of IROS'10, december 2010.

[8] S. Negahdaripour and P. Firoozfam, "An rov stereovision system for ship-hull inspection," IEEE J. of Oceanic Engineering, vol. 31(3), pp. 551-564, July 2006.

[9] A. Hogue, A. German, J. Zacher, and M. Jenkin, "Underwater 3d mapping: Experiences and lessons learned," Computer and Robot Vision, Canadian Conference, vol. 0, p. 24, 2006.

[10] R. Hartley and A. Zisserman, Multiple View Geometry in Computer Vision. Cambridge University Press, second ed., 2003.

[11] W. K. Pratt, Digital Image Processing: PIKS Inside. New York, NY, USA: John Wiley \& Sons, Inc., 2001.

[12] R. Szeliski, Computer Vision: Algorithms and Applications. 2010.

[13] T. Nicosevici, N. Gracias, S. Negahdaripour, and R. Garcia, "Efficient three-dimensional scene modeling and mosaicing," J. of Field Robot, vol. 26, pp. 759-788, October 2009.

[14] N. Pessel, J. Opderbecke, and M.-J. Aldon, "Camera self-calibration in underwater environment," in WSCG, 2003.

[15] J.-M. Lavest, G. Rives, and J.-T. Lapresté, "Underwater camera calibration," in 6th European Conf. on Computer Vision (ECCV), 2000.

[16] A. Meline, J. Triboulet, and B. Jouvencel, "A camcorder for 3d underwater reconstruction of archeological objects," in OCEANS'10, (Seattle Etats-Unis), pp. 1-9, MTS/IEEE, 2010.

[17] Z. Zhang, "A flexible new technique for camera calibration," IEEE Transactions on Pattern Analysis and Machine Intelligence, vol. 22, pp. 1330-1334, 2000.

[18] C. Harris and M. Stephens, "A combined corner and edge detection," in The Fourth Alvey Vision Conference, pp. 147-151, 1988.

[19] D. G. Lowe, "Distinctive image features from scale-invariant keypoints," Int. J. Comput. Vision, vol. 60, no. 2, pp. 91-110, 2004.

[20] M. A. Fischler and R. C. Bolles, "Random sample consensus: a paradigm for model fitting with applications to image analysis and automated cartography," Commun. ACM, vol. 24, pp. 381-395, 1981.

[21] R. Hartley, "In defence of the 8-points algorithm," IEEE Trans. On Pattern Analysis and Machine Intelligence, vol. 19(6), pp. 580-593, June 1997.

[22] K. Oliver, W. Hou, and S. Wang, "Image feature detection and matching in underwater conditions," in Ocean Sensing and Monitoring II - SPIE 7678, 2010.

[23] A. Sarafraz, S. Negahdaripour, and Y. Schechner, "Performance assessment in solving the correspondence problem in underwater stereo imagery," in OCEANS'10, (Seattle Etats-Unis), MTS/IEEE, 2010.

[24] W. Hou, "A simple underwater imaging model," Optics letters, vol. 34, no. 14, pp. 2688-2690, 2009.

[25] J. S. Jaffe, "Monte carlo modeling of underwater-image formation: validity of the linear and small-angle approximations," Appl. Opt., vol. 34, pp. 5413-5421, Aug 1995.

[26] R. Bandyopadhyay, A. S. Gittings, S. S. Suh, P. K. Dixon, and D. J. Durian, "Speckle-visibility spectroscopy: A tool to study time-varying dynamics," Review of Scientific Instruments, vol. 76, no. 9, pp. 93-110, 2005.

[27] R. Adrian and J. Westerweel, Particle Image Velocimetry. Cambridge University Press, 2010.

[28] R. Schettini and S. Corchs, "Underwater image processing: state of the art of restoration and image enhancement methods," EURASIP J. Adv. Signal Process, vol. 2010, pp. 14:1-14:7, January 2010. 